\section{Hábito de assistir à televisão e sua relação com a alimentação: resultados do período de 2006 a 2014 em capitais brasileiras}

\author{
Watching TV and eating habits: the results from \\ 2006 to 2014 in Brazilian state capitals
}

\author{
Hábito de ver televisión y su relación con la \\ alimentación: resultados del período de \\ 2006 a 2014 en capitales brasileñas
}

Emanuella Gomes Maia 1 Fernanda Mendes Dias Gomes 1 Marana Hauck Alves 1 Yara Rubia Huth 1 Rafael Moreira Claro ${ }^{1}$

\begin{abstract}
Resumo
O objetivo foi analisar a evolução da frequência do hábito de assistir à TV no Brasil e identificar a associação entre este hábito e o consumo alimentar na população adulta do Brasil entre os anos de 2006 e 2014. Dados do sistema de Vigilância de Fatores de Risco e Proteção para Doenças Crônicas por Inquérito Telefônico (VIGITEL) entre os anos de 2006 e 2014 foram utilizados. O hábito diário de assistir à TV e o consumo alimentar de frutas, hortaliças, feijão, carnes, leite, refrigerantes elou bebidas adoçadas foram analisados ao longo dos anos, sendo sua associação investigada por meio de modelos de regressão. A frequência de adultos referindo assistir à televisão por ao menos 3 horas por dia não variou significativamente ao longo dos anos, porém, nestes indivíduos, foi possível verificar um menor consumo de alimentos saudáveis, e maior, daqueles não saudáveis. Essa situação foi verificada em ambos os sexos e em todas as faixas de idade e escolaridade pesquisadas. O hábito de assistir à TV está associado ao consumo alimentar não saudável.
\end{abstract}

Estilo de Vida Sedentário; Sobrepeso; Obesidade; Consumo de Alimentos

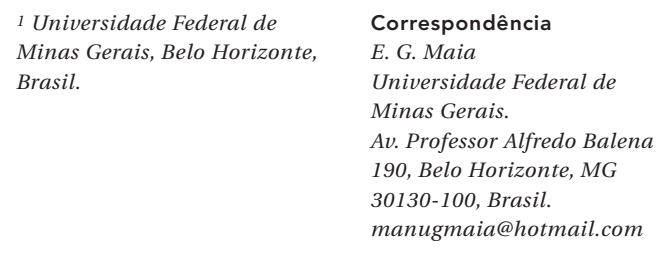

1 Universidade Federal de Minas Gerais, Belo Horizonte, Brasil.

\author{
Correspondência \\ E. G. Maia \\ Universidade Federal de \\ Minas Gerais. \\ Av. Professor Alfredo Balena \\ 190, Belo Horizonte, MG \\ 30130-100, Brasil. \\ manugmaia@hotmail.com
}




\section{Introdução}

O ganho excessivo de peso e a obesidade são os maiores problemas de nutrição em saúde pública da atualidade, atingindo uma parcela expressiva da população mundial 1 . No Brasil, dados da Pesquisa de Orçamentos Familiares (POF) de 2008/2009 mostraram que o excesso de peso (IMC $\geq 25 \mathrm{~kg} / \mathrm{m}^{2}$ ) já atingia $49 \%$ da população adulta, e que $14,8 \%$ dos brasileiros eram considerados obesos (IMC $\left.\geq 30 \mathrm{~kg} / \mathrm{m}^{2}\right)^{2}$. Além de ser por si só uma doença ${ }^{3}$, a obesidade é também um importante fator de risco para o desenvolvimento de diversas doenças e agravos não transmissíveis (DANT), como hipertensão arterial, dislipidemia e diabetes mellitus tipo II 1 . No ano de 2008, as DANT foram responsáveis por aproximadamente $72 \%$ dos óbitos ocorridos no Brasil e $63 \%$ em todo o mundo 4 .

O hábito de assistir à televisão por períodos prolongados de tempo se encontra entre os determinantes centrais desse quadro $5,6,7,8$, por simultaneamente promover o consumo alimentar não saudável e o comportamento sedentário 7,8. Desde a sua popularização, na metade do último século, a televisão tornou-se o passatempo preferido de populações tanto de países desenvolvidos quanto daqueles em desenvolvimento 9 . Dados da população estadunidense apontavam que, no ano de 2006, os domicílios daquele país passavam, em média, 8 horas ao dia com televisores ligados, com um tempo médio em frente à TV de 4,5 horas/dia por morador 10 . Ainda que não se conheça detalhadamente o hábito de assistir à televisão da população brasileira, sabe-se que, em 2014, cerca de um a cada quatro adultos residentes nas capitais dos estados e Distrito Federal refere assistir à TV por mais de três horas por dia $(25,3 \%) 11$.

Evidências indicam que o tempo gasto em frente à TV pode se relacionar ao ganho excessivo de peso por meio de três mecanismos: (i) o tempo gasto assistindo à TV implica a redução do tempo disponível para a realização da atividade física 12,13; (ii) indivíduos são encorajados a consumir alimentos de forma excessiva e desatenta enquanto assistindo à TV 7,12,14; e (iii) a exposição à publicidade de alimentos induz ao consumo dos produtos anunciados, sendo estes frequentemente não saudáveis 12,15.

Apesar da influência exercida pelo hábito de assistir à TV sobre o consumo alimentar identificada em países desenvolvidos, essencialmente entre crianças e adolescentes $16,17,18$, pouco se conhece sobre esta relação em populações adultas e em grandes amostras da população de países em desenvolvimento. Evidências disponíveis para países desenvolvidos indicam a presença tanto de relação direta entre o hábito de assistir à TV por longos períodos e o índice de massa corporal (IMC) de adultos (em estudos transversais 19,20 e longitudinais 21) quanto de relação inversa ao nível de atividade física 21 . Assim, por conhecer pouco a realidade da população adulta em países em desenvolvimento, os objetivos deste trabalho são analisar a evolução da frequência do hábito de assistir à televisão no Brasil e identificar a associação entre este hábito e o consumo alimentar na população adulta do Brasil entre os anos de 2006 e 2014.

\section{Métodos}

\section{Amostragem e coleta dos dados}

Estudo realizado com dados do sistema de Vigilância de Fatores de Risco e Proteção para Doenças Crônicas por Inquérito Telefônico (VIGITEL) entre os anos de 2006 e 2014. Trata-se de inquérito de base populacional que investiga fatores de risco e proteção para doenças crônicas não transmissíveis, entre eles excesso de peso, consumo alimentar e de bebidas alcoólicas, hábito de fumar, sedentarismo, nível de atividade física e prevenção de câncer 11. O VIGITEL entrevista, anualmente, amostras probabilísticas da população de adultos ( $\geq 18$ anos) que residem em domicílios com, ao menos, uma linha de telefone fixo, nas 26 capitais de estado do país e no Distrito Federal 11. Aproximadamente 2.000 entrevistas são realizadas em cada cidade, a cada ano (excepcionalmente, nos anos de 2012 e 2014 o número mínimo de entrevistas por cidade de realização do sistema foi reduzido para 1.500), totalizando aproximadamente 470 mil entrevistas no período de 2006-2014 11,22,23,24,25,26,27,28,29. Mais detalhes sobre o processo de amostragem empregado pelo VIGITEL podem ser obtidos nos relatórios anuais de divulgação dos dados do Sistema $11,22,23,24,25,26,27,28,29$

Todas as estimativas do VIGITEL são ponderadas para que representem o conjunto total da população adulta de cada cidade. $\mathrm{O}$ peso atribuído inicialmente a cada indivíduo entrevistado consiste na multiplicação de dois fatores: o inverso do número de linhas telefônicas e o número de adultos no domicílio do entrevistado. Um segundo peso, denominado peso pós-estratificação, tem como objetivo igualar a composição sociodemográfica da população servida por linhas telefônicas domiciliares à composição sociodemográfica da população adulta total de cada cidade no ano do levantamento ${ }^{11}$. 


\section{Organização dos dados}

O hábito de assistir à TV foi inicialmente aferido - no período entre 2006 e 2009 - por meio de três perguntas: “ $\mathrm{O}(\mathrm{a}) \mathrm{sr}$ (a) costuma assistir à televisão todos os dias? (Sim/Não)"; "Quantos dias por semana o(a) sr(a) costuma assistir à televisão? (5 ou mais, 3-4, 1-2, não costuma assistir à televisão)"; e "Quantas horas por dia o(a) $\operatorname{sr}($ a) costuma assistir à televisão? (< 1 hora, entre 1 e 2, entre 2 e 3 , entre 3 e 4 , entre 4 e 5 , entre 5 e 6 horas, mais de 6 horas)". Dada a alta frequência de indivíduos referindo assistir à televisão todos os dias (superior a 90\%), a partir de 2010, apenas a questão referente ao hábito diário (em horas/dia) foi mantida no questionário. Para as análises do presente estudo, o número de horas por dia assistindo à TV foi dicotomizado adotando-se como ponto de corte três horas diárias, seguindo a definição adotada pelo VIGITEL 11 para identificar os indivíduos com hábito de assistir à televisão por períodos prolongados (Tabela 1).

Entre as informações sobre consumo alimentar disponíveis, investigou-se o consumo recomendado de frutas e hortaliças (consumo de frutas e hortaliças pelo menos 5 vezes por dia em 5 ou mais dias da semana), consumo regular (em 5 ou mais dias da semana) de frutas e hortaliças, consumo de suco de frutas natural ao menos um dia da semana (disponíveis em: 2008-2014), consumo regular de frutas e de hortaliças isoladamente (disponíveis em: 2006-2014), consumo regular de feijão, consumo de carnes com excesso de gordura ao menos um dia da semana (carne vermelha com gordura e frango com pele), de leite com teor integral de gordura ao menos um dia da semana e consumo regular de refrigerantes (disponível 2007-2014), além de refrigerantes adoçados com adoçante calórico (disponíveis em: 2007-2011). Os indicadores de consumo regular (em 5 ou mais dias da semana) ou semanal (em ao menos um dia da semana) dos diversos alimentos são definidos com base em questões no seguinte formato: "Em quantos dias da semana o(a) sr(a) costuma comer [nome do grupo de alimentos]? (1-2 dias/semana, 3-4 dias/semana, 5-6 dias/semana, todos os dias, quase nunca, nunca)". Já o indicador de consumo recomendado de frutas e hortaliças considera o consumo de uma fruta ou de um suco de fruta como equivalente a uma porção, limitando-se em três o número máximo de porções diárias computado para frutas, e em um o número máximo para sucos. No caso de hortaliças, computou-se um número máximo de quatro porções diárias, situação que caracteriza indivíduos que informam o hábito de consumir saladas de hortaliças cruas no almoço e no jantar, e verduras e legumes cozidos também no almoço e no jantar. A recomendação para o consumo de frutas e hortaliças foi considerada alcançada quando o indivíduo referia o consumo destes alimentos em pelo menos cinco dias da semana, e quando a soma das porções consumidas diariamente destes alimentos totalizava pelo menos cinco (Tabela 1).

Um conjunto de variáveis sociodemográficas complementa a análise: sexo (masculino e feminino), idade (seis faixas de idade: 18-24, 25-34, 35-44, 45-54, 55-64 e $\geq 65$ anos) e escolaridade (três faixas de escolaridade: 0-8, 9-11 e $\geq 12$ anos de estudos). Essas variáveis foram utilizadas como variáveis de confundimento na investigação da associação entre o hábito de assistir à televisão por períodos prolongados e os indicadores de consumo alimentar.

\section{Análise dos dados}

A variação temporal na frequência do hábito de assistir à televisão por períodos prolongados $(\geq$ 3 horas/dia) foi investigada para o conjunto da população adulta das 27 cidades cobertas pelo Sistema e segundo sexo. O significado estatístico da tendência do indicador no período foi avaliado por meio de modelo de regressão linear, tendo como desfecho (variável dependente) a frequência do hábito de assistir à televisão por períodos prolongados e o ano do levantamento como variável explanatória (expresso como variável contínua). $\mathrm{O}$ coeficiente de regressão do modelo indica a taxa média anual, expressa em pontos percentuais (pp) ao ano, de aumento ou diminuição do indicador no período. Considerou-se significativa a variação correspondente a um coeficiente de regressão estatisticamente diferente de zero (valor de $\mathrm{p} \leq 0,05$ ).

As estimativas anuais de todos os indicadores do Sistema foram ponderadas para representar, em cada ano, a composição sociodemográfica da população adulta residente no conjunto das 27 cidades. Para tanto, foram utilizados os fatores de ponderação associados a cada um dos indivíduos da amostra VIGITEL estudados em cada ano do período 2006-2014. A natureza do cálculo desses fatores possibilita que os dados sejam analisados tanto separados, por capital e ano, quanto agregados 11. Maiores informações sobre a criação dos fatores de ponderação do VIGITEL podem ser encontradas no relatório anual de divulgação dos dados do sistema 11.

A frequência do hábito de assistir à televisão por períodos prolongados e dos indicadores de consumo alimentar foi apresentada para o conjunto completo da população (2006-2014) e segundo estratificações de sexo, faixa de idade e nível de escolaridade. Modelos de regressão de 
Definições das variáveis de consumo alimentar e hábito de assistir à televisão. Vigilância de Fatores de Risco e Proteção para Doenças Crônicas por Inquérito Telefônico (VIGITEL), Brasil, 2006-2014

\begin{tabular}{|c|c|c|}
\hline Variáveis & Definição da variável & Ano \\
\hline \multicolumn{3}{|l|}{ Sedentarismo } \\
\hline Hábito de assistir à TV & Hábito de assistir à televisão três ou mais horas por dia. & 2006-2014 \\
\hline \multicolumn{3}{|l|}{ Consumo alimentar } \\
\hline Consumo recomendado de frutas e hortaliças & $\begin{array}{l}\text { Consumo de pelo menos cinco porções diárias de frutas e } \\
\text { hortaliças por dia. }\end{array}$ & $2008-2014$ \\
\hline Consumo regular de frutas e hortaliças & Consumo de frutas e hortaliças em cinco ou mais dias da semana. & 2008-2014 \\
\hline Consumo de suco de frutas naturais * & Consumo de suco de frutas naturais ao menos um dia da semana. & 2008-2014 \\
\hline Consumo regular de frutas e hortaliças isoladamente & $\begin{array}{l}\text { Consumo de frutas em cinco ou mais dias da semana. Consumo de } \\
\text { hortaliças em cinco ou mais dias da semana. }\end{array}$ & 2006-2014 \\
\hline Consumo regular de feijão & Consumo de feijão em cinco ou mais dias da semana. & $2007-2014$ \\
\hline Consumo de carnes com excesso de gordura & $\begin{array}{l}\text { Consumo de carnes com excesso de gordura (carne com gordura } \\
\text { aparente e/ou frango com pele) ao menos um dia da semana. }\end{array}$ & $2007-2014$ \\
\hline Consumo de leite com teor integral de gordura & $\begin{array}{l}\text { Consumo de leite com teor integral de gordura ao menos um dia } \\
\qquad \text { da semana. }\end{array}$ & $2007-2014$ \\
\hline Consumo regular de refrigerantes & $\begin{array}{l}\text { Consumo de refrigerante (ou refresco/suco artificial) com ou sem } \\
\text { açúcar em cinco ou mais dias da semana. }\end{array}$ & 2007-2014 \\
\hline Consumo de refrigerantes adoçados & $\begin{array}{l}\text { Consumo de refrigerante (ou refresco/suco artificial) com adoçante } \\
\text { calórico em cinco ou mais dias da semana. }\end{array}$ & $2007-2011$ \\
\hline
\end{tabular}

* O consumo de suco de frutas naturais foi utilizado para o cálculo do consumo regular e recomendado de frutas e hortaliças.

Poisson foram usados para a investigação do efeito do hábito de assistir à televisão por períodos prolongados sobre os indicadores de consumo alimentar estudados, ajustada para variáveis sociodemográficas (sexo, faixa de idade e nível de escolaridade). Os indicadores de consumo alimentar foram adotados (um a um) como desfecho (variável dependente), e o hábito de assistir à televisão por períodos prolongados (na forma de uma variável dicotômica) foi adotado como condição explanatória. Essa análise foi realizada para o conjunto dos dados analisados (2006-2014) sem considerar o ano de realização do inquérito.

O aplicativo Stata, versão 12.1 (StataCorp LP, College Station, Estados Unidos) foi utilizado para processar os dados e para executar todas as análises apresentadas neste estudo, sempre levando em conta o delineamento da amostra do VIGITEL.

\section{Resultados}

No período entre 2006 e 2014, verificou-se variação estatisticamente significativa $(\mathrm{p}<0,05)$ na composição sociodemográfica da população adulta residente nas capitais de estado e Distrito Federal, especialmente com relação ao aumento de sua idade e nível de escolaridade (Tabela 2). Enquanto a frequência de indivíduos com idades entre 18 e 24 anos diminuiu de 18,9\% para 15,6\%, a frequência de indivíduos nas faixas extremas de idade (45-54 anos, 55-64 e 65 anos ou mais) aumentou, de $15,8 \%$ para $17,1 \%$, de $10 \%$ para $11,8 \%$ e de $9,4 \%$ para $10,6 \%$, respectivamente. Da mesma forma, a frequência de indivíduos com escolaridade entre 0 e 8 anos diminuiu de 45,5\% para $35,9 \%$ e a frequência daqueles com escolaridade superior a 12 anos aumentou de $21,2 \%$ para 25,9\% (Tabela 2).

No período entre 2006 e 2014, a frequência de adultos residentes nas capitais de estado e Distrito Federal referindo assistir à televisão por ao menos 3 horas por dia não obteve variação estatisticamente significativa ( $\mathrm{p}>0,05)$, oscilando entre 28\% em 2006 e 25,3\% em 2014. Situação semelhante foi verificada em ambos os sexos e em todas as faixas de idade e escolaridade pesquisadas. De forma geral, o hábito de assistir à televisão foi mais frequente nas faixas extremas de idade e entre indivíduos com baixa (0-8 anos) e média (9-11 anos) escolaridades (Tabela 3).

No conjunto completo do período estudado, indivíduos que referiam assistir à TV diariamente por mais de três horas apresentaram menor frequência de indicadores de consumo alimen- 
Tabela 2

Distribuição * (\%) da população adulta ( $\geq 18$ anos) das capitais de estados brasileiros e Distrito Federal, segundo sexo, idade e escolaridade. Vigilância de Fatores de Risco e Proteção para Doenças Crônicas por Inquérito Telefônico (VIGITEL), Brasil, 2006-2014.

\begin{tabular}{|c|c|c|c|c|c|c|c|c|c|}
\hline \multirow[t]{2}{*}{ Variáveis } & \multicolumn{9}{|c|}{ Distribuição da população estudada (\%) } \\
\hline & 2006 & 2007 & 2008 & 2009 & 2010 & 2011 & 2012 & 2013 & 2014 \\
\hline \multicolumn{10}{|l|}{ Sexo } \\
\hline Masculino & 46,1 & 46,2 & 46,1 & 46,1 & 46,1 & 46,1 & 46,1 & 46,1 & 46,1 \\
\hline Feminino & 53,9 & 53,8 & 53,9 & 53,9 & 53,9 & 53,9 & 53,9 & 53,9 & 53,9 \\
\hline \multicolumn{10}{|l|}{ Idade (anos) } \\
\hline $18-24$ & 18,9 & 18,2 & 17,9 & 17,2 & 17,1 & 16,7 & 16,4 & 15,9 & 15,6 \\
\hline $25-34$ & 25,4 & 25,4 & 25,4 & 25,5 & 25,4 & 25,4 & 25,2 & 25,3 & 25,3 \\
\hline $35-44$ & 20,6 & 20,5 & 20,4 & 20,3 & 20,1 & 20,0 & 19,9 & 19,7 & 19,6 \\
\hline $45-54$ & 15,8 & 15,9 & 16,1 & 16,3 & 16,4 & 16,6 & 16,8 & 16,9 & 17,1 \\
\hline $55-64$ & 10,0 & 10,2 & 10,4 & 10,7 & 10,9 & 11,1 & 11,4 & 11,6 & 11,8 \\
\hline 65 ou mais & 9,4 & 9,8 & 9,8 & 10,0 & 10,1 & 10,2 & 10,4 & 10,5 & 10,6 \\
\hline \multicolumn{10}{|c|}{ Escolaridade (anos) } \\
\hline $0-8$ & 45,5 & 45,0 & 43,7 & 42,0 & 40,6 & 38,8 & 36,8 & 36,6 & 35,9 \\
\hline $9-11$ & 33,3 & 35,1 & 34,7 & 35,8 & 35,8 & 36,7 & 38,5 & 37,5 & 38,1 \\
\hline 12 ou mais & 21,2 & 19,8 & 21,6 & 22,2 & 23,5 & 24,5 & 24,7 & 25,9 & 25,9 \\
\hline
\end{tabular}

* Valores ajustados para equiparar a população total estimada de cada cidade para cada um dos anos de estudo. Maiores detalhes na seção Métodos.

Tabela 3

Percentual de adultos ( $\geq 18$ anos) nas capitais de estados brasileiros e Distrito Federal que refere assistir à televisão diariamente por 3 ou mais horas, segundo sexo, idade e escolaridade. Vigilância de Fatores de Risco e Proteção para Doenças Crônicas por Inquérito Telefônico (VIGITEL), Brasil, $2006-2014$.

\begin{tabular}{|c|c|c|c|c|c|c|c|c|c|c|c|}
\hline \multirow[t]{2}{*}{ Características } & \multicolumn{9}{|c|}{ \% de indivíduos assistindo à TV diariamente por 3 horas ou mais } & \multirow[t]{2}{*}{ Variação anual (\%) * } & \multirow[t]{2}{*}{ Valor de $p$ ** } \\
\hline & 2006 & 2007 & 2008 & 2009 & 2010 & 2011 & 2012 & 2013 & 2014 & & \\
\hline \multicolumn{12}{|l|}{ Sexo } \\
\hline Masculino & 26,1 & 24,4 & 23,0 & 22,4 & 29,0 & 27,0 & 26,5 & 28,1 & 25,8 & 0,4 & 0,2 \\
\hline Feminino & 29,6 & 28,0 & 26,0 & 25,4 & 25,8 & 25,0 & 26,3 & 29,0 & 24,9 & $-0,3$ & 0,3 \\
\hline \multicolumn{12}{|l|}{ Idade (anos) } \\
\hline $18-24$ & 30,5 & 32,0 & 25,1 & 25,5 & 29,5 & 28,7 & 28,7 & 29,5 & 25,3 & $-0,3$ & 0,4 \\
\hline $25-34$ & 26,0 & 25,3 & 24,6 & 23,8 & 27,7 & 26,5 & 26,7 & 27,9 & 26,1 & 0,3 & 0,2 \\
\hline $35-44$ & 26,2 & 23,4 & 21,5 & 22,1 & 23,1 & 22,8 & 23,6 & 26,3 & 22,9 & 0,0 & 1,0 \\
\hline $45-54$ & 26,3 & 24,0 & 23,5 & 22,5 & 24,8 & 24,0 & 23,6 & 26,5 & 24,1 & 0,0 & 1,0 \\
\hline $55-64$ & 28,3 & 26,0 & 26,9 & 24,4 & 30,2 & 25,8 & 28,3 & 30,7 & 25,2 & 0,1 & 0,8 \\
\hline 65 ou mais & 34,3 & 28,9 & 29,6 & 28,4 & 31,2 & 29,5 & 30,0 & 34,3 & 30,1 & 0,0 & 0,9 \\
\hline \multirow{2}{*}{\multicolumn{12}{|c|}{$\begin{array}{l}\text { Escolaridade } \\
\text { (anos) }\end{array}$}} \\
\hline & & & & & & & & & & & \\
\hline $0-8$ & 30,3 & 27,2 & 27,0 & 26,8 & 27,9 & 26,6 & 27,8 & 31,4 & 26,3 & 0,0 & 0,9 \\
\hline $9-11$ & 30,9 & 29,8 & 27,0 & 25,9 & 29,6 & 28,6 & 28,7 & 30,6 & 27,8 & $-0,1$ & 0,8 \\
\hline 12 ou mais & 18,2 & 18,2 & 16,0 & 15,7 & 22,5 & 20,9 & 20,8 & 21,6 & 20,3 & 0,6 & 0,1 \\
\hline Total & 28,0 & 26,3 & 24,6 & 24,0 & 27,3 & 25,9 & 26,4 & 28,6 & 25,3 & 0,0 & 0,9 \\
\hline
\end{tabular}

* Correspondente ao coeficiente da regressão linear do valor do indicador sobre o ano do levantamento;

** O significado estatístico da tendência do indicador no período foi avaliado por meio de modelo de regressão linear, tendo como desfecho (variável dependente) o valor do indicador (por exemplo, o percentual de homens que referem assistir à TV diariamente por três ou mais horas) e como variável explanatória o ano do levantamento, expresso como variável contínua. 
tar saudável e maior dos não saudáveis, quando comparados aos sem este hábito, independentemente de seu sexo (Tabelas 4 e 5). Por exemplo, o consumo regular de frutas e hortaliças ( $\geq 5$ dias/ semana) foi 4,6 pp mais elevado entre aqueles que referiam assistir à TV por menos de três horas ao dia (respectivamente, $35,1 \%$ e $30,6 \%$ ) enquanto que o consumo de refrigerantes seguiu em caminho contrário, sendo 6,7 pp mais elevado entre os indivíduos com o hábito de assistir à TV diariamente por três ou mais horas (respectivamente, $30,9 \%$ e $24,1 \%$ ) (Tabela 4 ). Ainda que a magnitude das variações tenha sido diferente entre os sexos, a tendência verificada para o conjunto completo da população foi também observada tanto entre os homens quanto entre as mulheres (Tabela 5).
Após ajuste para idade e escolaridade (obtido por meio de modelo de regressão múltipla de Poisson), todas as diferenças inicialmente identificadas permaneceram estatisticamente significativas $(\mathrm{p}<0,05)$ (Tabelas 4 e 5). Do conjunto de indicadores analisados, apenas o hábito de consumir feijão com regularidade ( $\geq 5 \mathrm{dias} / \mathrm{se}$ mana) e de consumir leite com teor integral de gordura foram semelhantes entre os grupos de indivíduos, tanto na comparação bruta quanto naquela ajustada segundo idade e escolaridade (Tabelas 4 e 5).

Frequência de indicadores de consumo alimentar relacionados a doenças crônicas não transmissíveis na população adulta ( $\geq 18$ anos) das capitais brasileiras e Distrito Federal, segundo hábito de assistir à televisão. Vigilância de Fatores de Risco e Proteção para Doenças Crônicas por Inquérito Telefônico (VIGITEL), Brasil, 2006-2014.

\begin{tabular}{|c|c|c|c|c|c|c|}
\hline \multirow[t]{2}{*}{ Indicadores de consumo alimentar } & \multicolumn{2}{|c|}{ Assiste à TV $\geq 3$ horas/dia } & \multirow[t]{2}{*}{ RP bruta * } & \multirow[t]{2}{*}{ Valor de p } & \multirow[t]{2}{*}{ RP ajustada ** } & \multirow[t]{2}{*}{ Valor de $p$} \\
\hline & Não (\%) & $\operatorname{Sim}(\%)$ & & & & \\
\hline \multicolumn{7}{|l|}{ Consumo recomendado ( $\geq 5$ vezes/dia) } \\
\hline 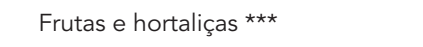 & 22,5 & 19,7 & 0,88 & 0,00 & 0,90 & 0,00 \\
\hline \multicolumn{7}{|l|}{ Consumo regular ( $\geq 5$ dias/semana) } \\
\hline Frutas e hortaliças *** & 35,1 & 30,6 & 0,87 & 0,00 & 0,89 & 0,00 \\
\hline Frutas \# & 57,8 & 55,3 & 0,96 & 0,00 & 0,96 & 0,00 \\
\hline Hortaliças \# & 50,8 & 45,5 & 0,90 & 0,00 & 0,91 & 0,00 \\
\hline Feijão \#\# & 66,2 & 66,9 & 1,01 & 0,06 & 1,00 & 0,49 \\
\hline Refrigerantes \#\# & 24,1 & 30,9 & 1,28 & 0,00 & 1,27 & 0,00 \\
\hline Refrigerantes adoçados \#\#\# & 22,2 & 29,0 & 1,30 & 0,00 & 1,27 & 0,00 \\
\hline 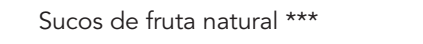 & 74,0 & 69,4 & 0,94 & 0,00 & 0,95 & 0,00 \\
\hline \multicolumn{7}{|l|}{ Consumo } \\
\hline Carne com excesso de gordura \#\# & 30,2 & 36,1 & 1,20 & 0,00 & 1,18 & 0,00 \\
\hline Leite integral \#\# & 55,0 & 55,1 & 1,00 & 0,74 & 0,99 & 0,31 \\
\hline
\end{tabular}

RP: razão de prevalência.

* RP bruta obtida em modelo de regressão de Poisson. Maiores detalhes na seção Métodos;

** RP ajustada por sexo, idade e escolaridade, obtida em modelo de regressão de Poisson. Maiores detalhes na seção Métodos;

*** Indicador disponível em 2008-2014;

\# Indicador disponível em 2006-2014;

\#\# Indicador disponível em 2007-2014;

\#\#\# Indicador disponível em 2007-2011. 
Frequência de indicadores de consumo alimentar relacionados a doenças crônicas não transmissíveis na população adulta ( $\geq 18$ anos) das capitais brasileiras e Distrito Federal, por sexo, segundo hábito de assistir à televisão. Vigilância de Fatores de Risco e Proteção para Doenças Crônicas por Inquérito Telefônico (VIGITEL), Brasil, 2006-2014.

\begin{tabular}{|c|c|c|c|c|c|c|}
\hline \multirow[t]{2}{*}{ Indicadores de consumo alimentar } & \multicolumn{2}{|c|}{ Assiste à TV $\geq 3$ horas/dia } & \multirow[t]{2}{*}{ RP bruta * } & \multirow[t]{2}{*}{ Valor de p } & \multirow{2}{*}{$\begin{array}{c}\text { RP ajustada } \\
\star \star \star\end{array}$} & \multirow[t]{2}{*}{ Valor de $\mathrm{p}$} \\
\hline & Não (\%) & $\operatorname{Sim}(\%)$ & & & & \\
\hline \multicolumn{7}{|l|}{ Homens } \\
\hline \multicolumn{7}{|l|}{ Consumo recomendado ( $\geq 5$ vezes/dia) } \\
\hline 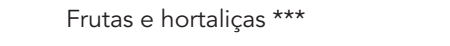 & 18,0 & 15,4 & 0,86 & 0,00 & 0,89 & 0,00 \\
\hline \multicolumn{7}{|l|}{ Consumo regular ( $\geq 5$ dias/semana) } \\
\hline 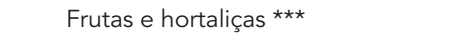 & 28,7 & 23,8 & 0,83 & 0,00 & 0,86 & 0,00 \\
\hline Frutas \# & 51,5 & 48,2 & 0,94 & 0,00 & 0,95 & 0,00 \\
\hline Hortaliças \# & 45,0 & 39,4 & 0,88 & 0,00 & 0,90 & 0,00 \\
\hline Feijão \#\# & 72,8 & 73,5 & 1,01 & 0,17 & 1,00 & 0,50 \\
\hline Refrigerantes \#\# & 27,6 & 35,5 & 1,28 & 0,00 & 1,26 & 0,00 \\
\hline Refrigerantes adoçados \#\#\# & 26,1 & 33,8 & 1,29 & 0,00 & 1,25 & 0,00 \\
\hline Sucos de fruta natural $* \star \star$ & 77,1 & 72,8 & 0,94 & 0,00 & 0,95 & 0,00 \\
\hline \multicolumn{7}{|l|}{ Consumo } \\
\hline Carne com excesso de gordura \#\# & 40,3 & 48,0 & 1,19 & 0,00 & 1,16 & 0,00 \\
\hline Leite integral \#\# & 57,4 & 57,3 & 1,00 & 0,82 & 0,99 & 0,27 \\
\hline \multicolumn{7}{|l|}{ Mulheres } \\
\hline \multicolumn{7}{|l|}{ Consumo recomendado ( $\geq 5$ vezes/dia) } \\
\hline 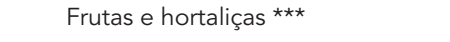 & 26,3 & 23,4 & 0,89 & 0,00 & 0,90 & 0,00 \\
\hline \multicolumn{7}{|l|}{ Consumo regular ( $\geq 5$ dias/semana) } \\
\hline 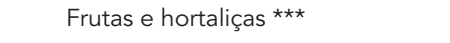 & 40,7 & 36,4 & 0,90 & 0,00 & 0,90 & 0,00 \\
\hline Frutas \# & 63,3 & 61,2 & 0,97 & 0,00 & 0,97 & 0,00 \\
\hline Hortaliças \# & 55,9 & 50,6 & 0,91 & 0,00 & 0,92 & 0,00 \\
\hline Feijão \#\# & 60,6 & 61,3 & 1,01 & 0,12 & 1,00 & 0,75 \\
\hline Refrigerantes \#\# & 21,1 & 27,0 & 1,28 & 0,00 & 1,28 & 0,00 \\
\hline Refrigerantes adoçados \#\#\# & 18,9 & 24,9 & 1,32 & 0,00 & 1,30 & 0,00 \\
\hline Sucos de fruta natural $\star \star \star$ & 71,4 & 66,5 & 0,93 & 0,00 & 0,94 & 0,00 \\
\hline \multicolumn{7}{|l|}{ Consumo } \\
\hline Carne com excesso de gordura \#\# & 21,4 & 26,0 & 1,21 & 0,00 & 1,20 & 0,00 \\
\hline Leite integral \#\# & 52,9 & 53,3 & 1,01 & 0,40 & 1,00 & 0,78 \\
\hline
\end{tabular}

RP: razão de prevalência.

* RP bruta obtida em modelo de regressão de Poisson. Maiores detalhes na seção Métodos;

** RP ajustada por sexo, idade e escolaridade, obtida em modelo de regressão de Poisson. Maiores detalhes na seção Métodos;

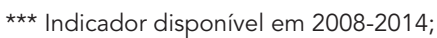

\# Indicador disponível em 2006-2014;

\#\# Indicador disponível em 2007-2014;

\#\#\# Indicador disponível em 2007-2011.

\section{Discussão}

Com base na coleta sistemática de dados realizada pelo VIGITEL entre os anos de 2006 e 2014, em uma amostra de cerca de 450 mil domicílios representativa da população adulta das 26 capitais dos estados brasileiros e Distrito Federal, foi possível analisar a evolução do hábito de assistir à televisão por período prolongado ( $\geq 3$ horas/dia) no país e sua associação com indicadores de consumo alimentar relacionados com doenças crônicas não transmissíveis (DCNT). No conjunto completo do período estudado (20062014), verificou-se estabilidade na proporção de indivíduos que referiam assistir à televisão diariamente por 3 ou mais horas, oscilando entre $28 \%$ e 25,3\% (alteração estatisticamente não significativa). Cenário semelhante foi encontra- 
do em ambos os sexos e todas as faixas de idade e escolaridade.

De forma geral, o hábito de assistir à televisão diariamente por 3 horas ou mais esteve associado a um pior consumo alimentar. Indivíduos que referiam esse hábito tenderam a apresentar maior frequência de indicadores do consumo alimentar não saudável (como o consumo regular de refrigerantes e carnes com excesso de gordura) e menor daqueles saudáveis (como o consumo regular de frutas e hortaliças). Essa situação foi observada na grande maioria dos indicadores analisados, tanto entre homens quanto entre mulheres. Apenas o hábito de consumir feijão com regularidade ( $\geq 5$ dias/semana) e de consumir leite com teor integral de gordura foram semelhantes entre os grupos de indivíduos que referiam assistir à televisão por, ao menos, 3 horas por dia e aqueles que não o faziam.

Contudo, algumas limitações devem ser observadas quanto aos resultados do nosso trabalho. Por se apoiar em entrevistas telefônicas, o VIGITEL não se propõe a medir diretamente o hábito de assistir à televisão na população, baseando-se na condição referida pelos entrevistados. Além disso, o instrumento usado para mensurar esse hábito no VIGITEL difere daqueles geralmente utilizados em estudos sobre comportamento sedentário (como o IPAQ - Questionário Internacional de Atividade Física, versões curta e longa, e o GPAQ - Questionário de Atividade Física Global) 30. Informações autorreferidas são frequentemente utilizadas em grandes inquéritos sobre condições de saúde e estilo de vida 31,32 devido à simplicidade e baixo custo de sua obtenção junto a grandes amostras populacionais $33,34,35$ e, até o momento, tanto o indicador do hábito de assistir à TV por períodos prolongados quanto os demais indicadores de prática de atividade física e de comportamentos sedentários apresentaram boa validade e reprodutibilidade 36,37 , indicando a adequação dos procedimentos empregados pelo VIGITEL. Adicionalmente, ainda que o questionário usado pelo VIGITEL não tenha sido inteiramente validado em uma grande amostra da população adulta das capitais de estados brasileiros e Distrito Federal, todos os estudos conduzidos até o momento com a população de São Paulo (2008) 36,38, Belém, Pará (2010) 39 e Belo Horizonte, Minas Gerais (2011 e 2013) 37,40 indicam a boa validade dos dados obtidos no Sistema. Cabe destacar ainda que tanto a utilização de técnicas pouco sofisticadas para aferição dos dados quanto à criação de um novo instrumento de coleta (em detrimento ao uso dos instrumentos mais comumente empregados para aferição de comportamentos sedentários na popu- lação) foram necessárias para que se pudesse entrevistar grande contingente de pessoas, em curto espaço de tempo e por meio de entrevista telefônica.

Além dessas questões, a restrição da amostra do VIGITEL aos indivíduos que têm telefone fixo nas capitais de estados brasileiros e no Distrito Federal pode também originar questionamentos quanto à validade dos dados. No entanto, desde a sua origem, o VIGITEL usa fatores de ponderação para ajustar as estimativas obtidas no inquérito telefônico de forma a corrigir as diferenças entre a população com e sem telefone, e assim permitir a extrapolação dos resultados para a população total das localidades avaliadas 11. Além disso, a metodologia utilizada para a construção dos fatores de ponderação do VIGITEL foi inteiramente atualizada no ano de 2012 28, possibilitando, entre outros, incorporar mudanças demográficas ocorridas na população estudada nas estimativas anuais do Sistema 28. Quanto à necessidade de inclusão no VIGITEL de entrevistas feitas por meio de telefones celulares, resultados de um estudo piloto conduzido em 2008 destacavam a pouca vantagem desta atualização metodológica frente ao seu alto custo 41

Por fim, deve-se ter cautela na interpretação dos resultados também devido ao curto período estudado (2006-2014). Por apresentar série histórica limitada (período máximo de nove anos), variações temporais que não tenham sido uniformes tendem a não ser identificadas, de modo que a identificação mais detalhada das tendências de evolução do indicador estudado passa necessariamente pela ampliação do período em estudo pelo Sistema 11.

Conforme exposto anteriormente, os dados coletados pelo VIGITEL são representativos da população adulta residindo nas capitais de estado do país e no Distrito Federal 11,22,23,24,25,26,27,28,29. Ainda que seja normal supor que a prevalência de indivíduos que referem assistir à televisão diariamente por 3 ou mais horas, identificada em nosso estudo, seja diferente daquela de outros domínios geográficos do país, acredita-se que a associação entre o hábito de assistir à TV por períodos prolongados e comportamentos prejudiciais à saúde seja realidade também em outros cenários urbanos do país, semelhantes às cidades investigadas no VIGITEL.

Diversos trabalhos na literatura foram dedicados a analisar o hábito de assistir à TV e suas repercussões para a saúde dos indivíduos adultos $5,7,8$, indicando que este hábito prediz não apenas o ganho de peso 20,21 , mas também morbidades como a síndrome metabólica 19 e o aumento da mortalidade entre homens e mulheres, principalmente quanto às causas 
cardiovasculares 7. Porém, poucos estudos analisaram essa relação com vistas, especialmente, ao consumo alimentar de adultos em países em desenvolvimento 42 . No Brasil, a maioria dos estudos realizados é dedicada a avaliar essas relações apenas em crianças ou adolescentes 43,44, ou restringem sua abordagem à análise da associação entre o hábito de assistir à TV e a prática de atividade física 45,46 .

O percentual de indivíduos que referiram assistir à televisão por três ou mais horas ao dia, nas capitais de estados brasileiros e Distrito Federal, manteve-se estável no período entre 2006 e 2014. Ainda que essa estabilidade possa ser vista como uma notícia positiva, ela não deve ser vista como uma proxy do comportamento sedentário no lazer. O aumento do acesso às atividades de lazer sedentário nos últimos 20 anos - como o uso doméstico de computadores e da internet, além da maior presença de vídeo games, celulares e tablets nos domicílios brasileiros - expôs a real concorrência à televisão na preferência dos indivíduos, de modo que uma medida acurada do tempo despendido em atividades sedentárias só possa ser acessada por meio da averiguação do tempo gasto em todas estas atividades. De todo modo, a televisão permanece sendo o passatempo dominante entre pessoas de todas as faixas etárias. No intuito de confirmar essa estabilidade, análises complementares foram realizadas para cada um dos anos. O número médio de horas assistindo à TV ao dia (e seu intervalo de 95\% de confiança - IC95\%) entre os indivíduos que referiam assisti-la por 3 ou mais horas ao dia e aqueles que referiam não fazê-lo foi calculado. Em ambos os casos não foram observadas mudanças estatisticamente significativas, com os valores se mantendo estáveis com o passar dos anos (cerca de 4,5 horas/dia e 1,5 hora/ dia, respectivamente).

Dados de um estudo envolvendo 28 países da Europa mostraram que a prevalência de jovens (11-15 anos) que assistiam à televisão por 4 ou mais horas ao dia variava entre $26,5 \%$ e $49,2 \% 47$, e um estudo realizado entre 1994 e 1996 nos Estados Unidos já apontava que quase dois a cada três americanos adultos (58,9\%) assistiam à televisão por duas horas ou mais ao dia 48 . A comparação de nossos achados com os de outros estudos deve ser realizada com cautela em função das diferenças nas características da população estudada, nos instrumentos utilizados para avaliação do consumo alimentar e horas diárias destinadas à TV, além dos pontos de corte empregados nas análises estatísticas. Nesse caso, a frequência de adultos que assistiam à televisão por períodos prolongados no estudo americano é necessariamente maior do que a do nosso es- tudo, pois o ponto de corte usado nesta análise foi menor (2 horas/dia).

Desde meados da década de 1990, a relação entre o hábito de ver TV e o ganho excessivo de peso já era conhecida 48 , tendo as evidências neste sentido se proliferado até o momento atual 5,17,49. Além disso, outros achados associam esse hábito ao menor nível de condicionamento físico 50 , dislipidemia 49 , pressão arterial elevada ${ }^{51}$, diabetes tipo II 52 e síndrome metabólica 19. De forma complementar, nossos resultados evidenciam, de forma inédita, a associação entre o hábito de ver TV por períodos prolongados e o consumo alimentar não saudável em adultos no país. Esses dados complementam os achados disponíveis até este momento, indicando a associação positiva entre o hábito de assistir à TV e o aumento da frequência de consumo e da quantidade total consumida de alimentos 53,54. Evidência obtida em estudo experimental demonstra ainda que o hábito de assistir à TV é ainda capaz de aumentar o consumo de alimentos de alta densidade energética 55 .

Dentre os possíveis mecanismos por meio dos quais o hábito de assistir à televisão influenciaria o consumo alimentar, destaca-se a capacidade da TV de estimular o consumo mesmo em indivíduos sem fome ${ }^{54}$. Assistir à TV é uma atividade distrativa que faz com que as pessoas ignorem sensações como a saciedade. Assim, quanto maior for a concentração do telespectador na atração, maior a propensão ao consumo de alimentos 56. Complementando esse cenário, a publicidade de alimentos direciona as escolhas em torno de produtos ultraprocessados de alta densidade energética e baixo teor nutricional 57,58,59. Cabe destacar, que entre os dez passos para uma alimentação saudável, a mais recente edição do Guia Alimentar para a População Brasileira recomenda que os indivíduos façam suas refeições em lugares calmos e tranquilos (longe de televisores) e que sejam críticos quanto ao conteúdo dos comerciais 60 .

A despeito da maior frequência do hábito de assistir à TV por períodos prolongados ter sido mais frequente nas faixas estremas de idades e entre pessoas na faixa inferior de escolaridade, altas proporções foram observadas em todos os cenários estudados. Aliado à associação entre esse hábito e o comportamento alimentar não saudável, fica evidente a necessidade de medidas destinadas a reduzir o número de horas gastas pela população vendo TV ou, até mesmo, a regulamentar a publicidade de alimentos não saudáveis nesse importante veículo de comunicação. Nesse contexto, algumas medidas são extremamente importantes para facilitar o acesso de indivíduos (principalmente os de menor 
nível socioeconômico) a equipamentos públicos de lazer nas grandes cidades brasileiras, tanto relacionadas à priorização do transporte coletivo socialmente inclusivo quanto à garantia da segurança, preservação e manutenção de espaços urbanos que convidem estes indivíduos ao convívio comunitário. Programas vêm sendo desenvolvidos em todo o território nacional no sentido de facilitar a adoção de atividades de lazer não sedentárias - como o Programa Academia da Cidade e a revitalização de praças e parques 4 - e diversos projetos estão sendo discutidos pelo governo federal, com o intuito de regulamentar a publicidade destinada aos grupos populacionais mais vulneráveis (como as crianças) 61,62, mas que ainda demandam muitos esforços para que haja avanços efetivos.

\section{Colaboradores}

E. G. Maia e R. M. Claro contribuíram na concepção e delineamento do estudo, análise e interpretação dos dados, redação e revisão do artigo. F. M. D. Gomes, M. H. Alves e Y. R. Huth colaboraram na análise e interpretação dos dados e revisão do artigo.

\section{Conclusão}

O presente estudo permitiu expor, de forma inédita, a evolução do hábito de assistir à TV por períodos prolongados em adultos que residem nas capitais de estado e Distrito Federal, no período entre 2006 e 2014. Não foram verificadas mudanças estatisticamente significativas (de aumento ou diminuição) em nenhum dos estratos populacionais de sexo, idade e escolaridade estudados. Contudo, esse hábito esteve associado ao consumo alimentar não saudável, ressaltando a necessidade de sua redução e o aumento da prática de atividades de lazer não sedentárias.

\section{Agradecimentos}

Os autores agradecem à Coordenação de Aperfeiçoamento de Pessoal de Nível Superior (Capes) pelo financiamento de bolsa de estudo. 


\section{Referências}

1. World Health Organization. Diet, nutrition and the prevention of chronic diseases. http://whqlibdoc. who.int/trs/who_trs_916.pdf (acessado em 10/ Jun/2015).

2. Instituto Brasileiro de Geografia e Estatística. Pesquisa de Orçamentos Familiares 2008/2009: análise do consumo alimentar pessoal no Brasil. http://biblioteca.ibge.gov.br/visualizacao/livros/ liv50063.pdf (acessado em 14/Jun/2015).

3. Prado WL, Lofrano MC, Oyama LM, Dâmaso AR. Obesity and inflammatory adipokines: practical implications for exercise prescription. Rev Bras Med Esporte 2009; 15:378-83.

4. Departamento de Análise de Situação de Saúde, Secretaria de Vigilância em Saúde, Ministério da Saúde. Plano de ações estratégicas para o enfrentamento das doenças crônicas não transmissíveis (DCNT) no Brasil 2011-2022. http://www.sbn.org. $\mathrm{br} /$ noticias/acoes estrategicas.pdf (acessado em 14/Jun/2015).

5. Hu FB, Li TY, Colditz GA, Willett WC, Manson JE. Television watching and other sedentary behaviors in relation to risk of obesity and type 2 diabetes mellitus in women. JAMA 2003; 289:1785-91.

6. Manios Y, Kourlaba G, Kondaki K, Grammatikaki E, Anastasiadou A, Roma-Giannikou E, et al. Obesity and television watching in preschoolers in Greece: The Genesis study. Obesity 2009; 17:2047-53.

7. Thorp AA, Owen N, Neuhaus M, Dunstan DW. Sedentary behaviors and subsequent health outcomes in adults a systematic review of longitudinal studies, 1996-2011. Am J Prev Med 2011; 41:207-15.

8. Marsh S, Ni Mhurchu C, Maddison R. The non-advertising effects of screen-based sedentary activities on acute eating behaviours in children, adolescents, and young adults. A systematic review. Appetite 2013; 71:259-73.

9. Vereeckn CA, Todd J, Roberts C, Mulvihill C, Maes L. Television viewing behaviour and associations with food habits in different countries. Public Health Nutr 2005; 9:244-50.

10. Nielsen Media research reports televisions popularity is still growing. http://www.prnewswire. $\mathrm{com} /$ news-releases/nielsen-media-researchreports-televisions-popularity-is-still-grow ing-57098217.html (acessado em 16/Jun/2015).

11. Departamento de Vigilância de Doenças e Agravos não Transmissíveis e Promoção da Saúde, Secretaria de Vigilância em Saúde, Ministério da Saúde. Vigitel Brasil 2014: vigilância de fatores de risco e proteção para doenças crônicas por inquérito telefônico. http://portalsaude.saude.gov.br/images/ pdf/2015/agosto/03/Vigitel-2014-final.pdf (acessado em 16/Jun/2015).

12. Council on Communications and Media. Children, adolescents, obesity, and the media. Pediatrics 2011; 128:201-8.

13. Lowry R, Michael S, Demissie Z, Kann L, Galuska DA. Associations of physical activity and sedentary behaviors with dietary behaviors among US high school students. J Obes 2015; 2015:876524.
14. Pearson N, Biddle SJH, Williams L, Worsley A, Crawford D, Ball K. Adolescent television viewing and unhealthy snack food consumption: the mediating role of home availability of unhealthy snack foods. Public Health Nutr 2012; 17:317-23.

15. Harris JL, Sarda V, Schwartz MB, Brownell KD. Redefining "child-directed advertising" to reduce unhealthy television food advertising. Am J Prev Med 2013; 44:358-64.

16. Danner, FW. A national longitudinal study of the association between hours of TV viewing and the trajectory of BMI growth among US children. J Pediatr Psychol 2008; 33:1100-7.

17. Morales-Suárez-Varela M, Ruso Julve C, Llopis González A. Comparative study of lifestyle: eating habits, sedentary lifestyle and anthropometric development in Spanish 5- to 15-yr-Olds. Iran J Public Health 2015; 44:486-94.

18. Zhou Z, Diao Q, Shao N, Liang Y, Lin L, Lei Y, et al. The frequency of unhealthy food advertising on mainland chinese television (TV) and children and adolescents' risk of exposure to them. PLoS One 2015; 10:e0128746.

19. Thorp AA, McNaughton SA, Owen N, Dunstan DW. Independent and joint associations of TV viewing time and snack food consumption with the metabolic syndrome and its components; a cross-sectional study in Australian adults. Int J Behav Nutr Phys Act 2013; 10:96.

20. Xie YJ, Stewart SM, Lam TH, Viswanath K, Chan SS. Television viewing time in Hong Kong adult population: associations with body mass index and obesity. PLoS One 2014; 9:e85440.

21. Gardner B, Iliffe S, Fox KR, Jefferis BJ, Hamer M. Sociodemographic, behavioural and health factors associated with changes in older adults' TV viewing over 2 years. Int J Behav Nutr Phys Act 2014; $11: 102$.

22. Departamento de Vigilância de Doenças e Agravos não Transmissíveis e Promoção da Saúde, Secretaria de Vigilância em Saúde, Ministério da Saúde. Vigitel Brasil 2006: vigilância de fatores de risco e proteção para doenças crônicas por inquérito telefônico. http://portalsaude.saude.gov.br/images/ pdf/2014/setembro/09/vigitel-2006-brasil.pdf (acessado em 16/Jun/2015).

23. Departamento de Vigilância de Doenças e Agravos não Transmissíveis e Promoção da Saúde, Secretaria de Vigilância em Saúde, Ministério da Saúde. Vigitel Brasil 2007: vigilância de fatores de risco e proteção para doenças crônicas por inquérito telefônico. http://portalsaude.saude.gov.br/images/ pdf/2014/setembro/09/vigitel-2007-Brasil.pdf (acessado em 16/Jun/2015).

24. Departamento de Vigilância de Doenças e Agravos não Transmissíveis e Promoção da Saúde, Secretaria de Vigilância em Saúde, Ministério da Saúde. Vigitel Brasil 2008: vigilância de fatores de risco e proteção para doenças crônicas por inquérito telefônico. http://portalsaude.saude.gov.br/images/ pdf/2014/setembro/09/vigitel-2008.pdf (acessado em 16/Jun/2015). 
25. Departamento de Vigilância de Doenças e Agravos não Transmissíveis e Promoção da Saúde, Secretaria de Vigilância em Saúde, Ministério da Saúde. Vigitel Brasil 2009: vigilância de fatores de risco e proteção para doenças crônicas por inquérito telefônico. http://portalsaude.saude.gov.br/ima ges/pdf/2014/setembro/09/vigitel-2009-web.pdf (acessado em 16/Jun/2015).

26. Departamento de Vigilância de Doenças e Agravos não Transmissíveis e Promoção da Saúde, Secretaria de Vigilância em Saúde, Ministério da Saúde. Vigitel Brasil 2010: vigilância de fatores de risco e proteção para doenças crônicas por inquérito telefônico. http://portalsaude.saude.gov.br/images/ pdf/2014/dezembro/23/VIGITEL-2010.pdf (acessado em 16/Jun/2015)

27. Departamento de Vigilância de Doenças e Agravos não Transmissíveis e Promoção da Saúde, Secretaria de Vigilância em Saúde, Ministério da Saúde. Vigitel Brasil 2011: vigilância de fatores de risco e proteção para doenças crônicas por inquérito telefônico. http://portalsaude.saude.gov.br/images/ pdf/2014/setembro/09/vigitel-2011.pdf (acessado em 16/Jun/2015).

28. Departamento de Vigilância de Doenças e Agravos não Transmissíveis e Promoção da Saúde, Secretaria de Vigilância em Saúde, Ministério da Saúde. Vigitel Brasil 2012: vigilância de fatores de risco e proteção para doenças crônicas por inquérito telefônico. http://portalsaude.saude.gov.br/images/ pdf/2014/setembro/09/vigitel-2012.pdf (acessado em 16/Jun/2015).

29. Departamento de Vigilância de Doenças e Agravos não Transmissíveis e Promoção da Saúde, Secretaria de Vigilância em Saúde, Ministério da Saúde. Vigitel Brasil 2013: vigilância de fatores de risco e proteção para doenças crônicas por inquérito telefônico. http://portalsaude.saude.gov.br/images/ pdf/2014/dezembro/09/Vigitel-2013.pdf (acessado em 16/Jun/2015).

30. Healy GN, Clark BK, Winkler EA, Gardiner PA, Brown WJ, Matthews CE. Measurement of adults' sedentary time in population-based studies. Am J Prev Med 2011; 41:216-27.

31. Instituto Brasileiro de Geografia e Estatística. Pesquisa Nacional de Saúde Escolar (PeNSE), 2012. http://portalsaude.saude.gov.br/images/pdf/2014/ dezembro/09/PeNSE-2012.pdf (acessado em 18/ Jun/2015)

32. Centers for Disease Control and Prevention. Behavioral Risk Factor Surveillance System - BRFSS http://www.cdc.gov/brfss/questionnaires/pdfques/2014_BRFSS.pdf (acessado em 16/Jun/2015).

33. Monteiro CA, de Moura EC, Jaime PC, Lucca A, Florindo AA, Figueiredo ICR, et al. Monitoramento de fatores de risco para doenças crônicas por entrevistas telefônicas. Rev Saúde Pública 2005; 39:47-57.
34. Moura EC, Morais Neto OL, Malta DC, Moura L, Silva NN, Bernal R, et al. Vigilância de fatores de risco para doenças crônicas por inquérito telefônico nas capitais dos 26 estados brasileiros e no Distrito Federal (2006). Rev Bras Epidemiol 2008; 11:20-37.

35. Szolnokin G, Hoffmann D. Online, face-to-face and telephone surveys - Comparing different sampling methods in wine consumer research. Wine Economics and Policy 2013; 2:57-66.

36. Monteiro CA, Florindo AA, Claro RM, Moura EC Validade de indicadores de atividade física e sedentarismo obtidos por inquérito telefônico. Rev Saúde Pública 2008; 42:575-81.

37. Moreira AD. Validade e reprodutibilidade de questionário de atividade física do sistema de vigilância de fatores de risco e proteção para doenças crônicas por inquérito telefônico [Dissertação de Mestrado]. Belo Horizonte: Universidade Federal de Minas Gerais; 2013.

38. Monteiro CA, Moura EC, Jaime PC, Claro RM. Validade de indicadores do consumo alimentos e bebidas obtidos por inquérito telefônico. Rev Saúde Pública 2008; 42:582-9.

39. Neves ACMD, Gonzaga LAA, Martens IBG, Moura EC. Validação de indicadores do consumo de alimentos e bebidas obtidos por inquérito telefônico em Belém, Pará, Brasil. Cad Saúde Pública 2010; 26:2379-88.

40. Ferreira AD, César CC, Malta DC, Andrade ACS, Ramos CGC, Proietti FA, et al. Validade de estimativas obtidas por inquérito telefônico: comparação entre VIGITEL 2008 e Inquérito Saúde em Beagá. Rev Bras Epidemiol 2011; 14:16-30.

41. Moura EC, Claro RM, Bernal R, Ribeiro J, Malta DC, Marais Neto O. A feasibility study of cell phone and landline phone interviews for monitoring of risk and protection factors for chronic diseases in Brazil. Cad Saúde Pública 2011; 27:277-86.

42.. Moraes SA, Beltrán RJ, Mondini L, Freitas ICM. Prevalência de sobrepeso e obesidade e fatores associados em escolares de área urbana de Chilpancingo, Guerrero, México, 2004. Cad Saúde Pública 2006; 22:1289-301.

43. Fernandes RA, Christofaro DG, Casonatto J, Kawaguti SS, Ronque ER, Cardoso JR, et al. Crosssectional association between healthy and unhealthy food habits and leisure physical activity in adolescents. J Pediatr (Rio J.) 2011; 87:252-6.

44. Camelo LV, Rodrigues JFC, Giatti L, Barreto SM. Lazer sedentário e consumo de alimentos entre adolescentes brasileiros: Pesquisa Nacional de Saúde do Escolar (PeNSE), 2009. Cad Saúde Pública 2012; 28:2155-62.

45. Hallal PC, Knuth AG, Cruz DKA, Mendes MI, Malta DC. Physical activity practice among Brazilian adolescentes. Ciênc Saúde Coletiva 2010; 15:3035-42. 
46. Knuth AG, Malta DC, Dumith SC, Pereira CA, Morais Neto OL. Practice of physical activity and sedentarism among Brazilians: results of the National Household Sample Survey - 2008. Ciênc Saúde Coletiva 2011; 16:3697-705.

47. Currie C, Roberts C, Morgan A, Smith R, Settertobulte W, Samdal O, et al. Young people's health in context. Health Behaviour in School-Aged Children (HBSC) Study. International Report from the 2001/2002 Survey. http://www.euro.who.int/_da ta/assets/pdf_file/0008/110231/e82923.pdf (acessado em 18/Jun/2015).

48. Bowman SA. Television-viewing characteristics of adults: correlations to eating practices and overweight and health status. Prev Chronic Dis 2006; 3:A38.

49. Salmon J, Bauman A, Crawford D, Timperio A, Owen $\mathrm{N}$. The association between television viewing and overweight among Australian adults participating in varying levels of leisure-time physical activity. Int J Obes Relat Metab Disord 2000; 24:600-6.

50. Tucker LA. Television viewing and physical fitness in adults. Res Q Exerc Sport 1990; 61:315-20.

51. Jakes RW, Day N, Khaw KT, Luben R, Oakes S, Welch A, et al. Television viewing and low participation in vigorous recreation are independently associated with obesity and markers of cardiovascular disease risk: EPIC-Norfolk population-based study. Eur J Clin Nutr 2003; 57:1089-96.

52. Dunstan DW, Salmon J, Owen N, Armstrong T, Zimmet PZ, Welborn TA, et al. Associations of TV viewing and physical activity with the metabolic syndrome in Australian adults. Diabetologia 2005; 48:2254-61.

53. Stroebele N, Castro JM. Television viewing is associated with an increase in meal frequency in humans. Appetite 2004; 42:111-3.
54. Blass EM, Anderson DR, Kirkorian HL, Pempek TA, Price I, Koleini MF. On the road to obesity: television viewing increases intake of high-density foods. Physiol Behav 2006; 88:597-604.

55. Bellisle F, Dalix AM, Slama G. Non food-related environmental stimuli induce increased meal intake in healthy women: comparison of television viewing versus listening to a recorded story in laboratory settings. Appetite 2004; 43:175-80.

56. Wansink B, Park SB. At the movies: how external cues and perceived taste impact consumption volume. Food Qual Prefer 2001; 12:69-74.

57. Story M, French S. Food advertising and marketing directed at children and adolescents in the US. Int J Behav Nutr Phys Act 2004; 1:3.

58. Monteiro CA, Cannon G. The impact of transnational "big food" companies on the South: a view from Brazil. PLoS Med 2012; 9:e1001252.

59. Marques CSM, Martins HP, Santos LC. Análise dos alimentos anunciados durante a programação infantil em emissoras de canal aberto no Brasil. Rev Bras Epidemiol 2013; 16:976-83.

60. Ministério da Saúde. Guia alimentar para a população brasileira, 2014. http://portalsaude.saude. gov.br/images/pdf/2014/novembro/05/Guia-Ali mentar-para-a-pop-brasiliera-Miolo-PDF-Inter net.pdf (acessado em 16/Jun/2015).

61. Monteiro CA, Castro IRR. Por que é necessário regulamentar a publicidade de alimentos. Ciênc Cult (São Paulo) 2009; 61:56-9.

62. Henriques P, Dias PC, Burlandy L. A regulamentação da propaganda de alimentos no Brasil: convergências e conflitos de interesses. Cad Saúde Pública 2014; 30:1219-28. 


\section{Abstract}

The objectives were to analyze trends in TV watching in Brazil and to identify the association between this habit and food consumption in the Brazilian adult population from 2006 to 2014. Data were obtained from the Surveillance System for Risk and Protective Factors for Chronic Illnesses Using a Telephone Survey (VIGITEL) for the years 2006 to 2014. The daily habit of watching TV and consumption of fruits, vegetables, beans, meat, milk, sodas, and/or sweetened beverages were analyzed over the period, and their association was investigated using regression models. The proportion of adults that reported watching more than three hours of TV per day did not vary significantly over the years, but these individuals showed declining consumption of healthy foods and increasing consumption of unhealthy foods. This situation was observed in both sexes and in all age and schooling brackets. The habit of watching TV is associated with unhealthy eating.

Sedentary Lifestyle; Overweight; Obesity;

Food Consumption

\section{Resumen}

El objetivo fue analizar la evolución de la frecuencia del hábito de ver TV en Brasil e identificar la asociación entre el hábito de ver TV y el consumo alimenticio en la población adulta de Brasil entre los años de 2006 y 2014. Se utilizaron datos del sistema de Vigilancia de Factores de Riesgo y Protección para Enfermedades Crónicas por Encuesta Telefónica (VIGITEL) entre los años 2006 y 2014. El hábito diario de ver TVy el consumo alimenticio de frutas, hortalizas, frijoles, carnes, leche, refrescos y/o bebidas azucaradas fueron analizados a lo largo de los años, siendo su asociación investigada mediante modelos de regresión. La frecuencia de adultos informando que ven televisión por lo menos 3 horas al día no varió significativamente a lo largo de los años, no obstante, en estos individuos, fue posible verificar un menor consumo de alimentos saludables, y mayor que el de aquellos no saludables. Esta situación fue verificada en ambos sexos y en todas las franjas de edad y escolaridad investigadas. El hábito de ver TV está asociado a un consumo alimenticio no saludable.

Estilo de Vida Sedentario; Sobrepeso; Obesidad; Consumo de Alimentos
Recebido em 01/Jul/2015

Versão final reapresentada em 18/Nov/2015 Aprovado em 22/Fev/2016 